\title{
THE COHOMOLOGY OF SEMISIMPLE LIE ALGEBRAS WITH COEFFICIENTS IN A VERMA MODULE( $\left.{ }^{\mathbf{1}}\right)$ \\ BY
}

FLOYD L. WILLIAMS

\begin{abstract}
The structure of the cohomology of a complex semisimple Lie algebra with coefficients in an arbitrary Verma module is completely determined. Because the Verma modules are infinite-dimensional, the cohomology need not vanish (as it does for nontrivial finite-dimensional modules). The methods presented exploit the homological machinery of Cartan-Eilenberg [3]. The results of [3], when applied to the universal enveloping algebra of a semisimple Lie algebra and when coupled with key results of Kostant [12], Hochschild-Serre [9], yield the basic structure theorem-Theorem 4.19. Our results show, incidently, that an assertion of $\mathbf{H}$. Kimura, Theorem 2 of [13] is false. A counterexample is presented in 86.
\end{abstract}

1. Introduction. Let $g$ be a finite-dimensional complex semisimple Lie algebra, let $b$ be a Borel subalgebra of $g$, and let $U g, U b$ denote the universal algebras of $g, b$ respectively. If $W$ is a finite-dimensional irreducible left $U b$ module ( $W$ is one-dimensional by Lie's theorem) then $U_{g} \otimes_{U b} W$ is a left $U g$ module. Here we regard $U g$ as a $(U g, U b)$ bimodule; i.e. $U g$ is a left $U g$, right $U b$ module with commuting action because of the associative law. The "induced" modules $U g \otimes_{U b} W$, where $W$ varies, are called Verma modules [1], [14], [15]. They enjoy many pleasant properties and are beginning to play an increasingly important role in the unitary representation theory of semisimple Lie groups.

In this paper we give the structure of the cohomology $H^{k}\left(g, U g \otimes_{U b} W\right)$ of $g$ with coefficients in $U g \otimes_{U b} W$ for all $k$ and for all $W$. The main result is Theorem 4.19. We compute the cohomology of the dual module $\left(U g \otimes_{U b} W\right)^{*}=\operatorname{Hom}_{U b}\left(U g, W^{*}\right)$ as well (Theorem 4.15) and, in fact, we obtain Theorem 4.19 by applying a duality theorem (Theorem 3.4 ) which is the slight independent interest.

The modules $U g \otimes_{U b} W$ are infinite-dimensional of course and, as our results show, the cohomology spaces $H^{k}\left(g, U g \otimes_{U b} W\right)$ can very well be nonzero. In contrast, the classical Whitehead Lemmas assert that when $V$ is a

Received by the editors July $19,1976$.

AMS (MOS) subject classifications (1970). Primary 18H25, 17B10.

Key words and phrases. Complex semisimple Lie algebra, Lie algebra cohomology, Verma module, highest weight, spectral sequence.

(')Research supported by NSF grant MPS 70-01864-A04. 
finite-dimensional nontrivial $g$ module, the cohomology spaces $H^{k}(g, V)$ are always zero [11]. Even though the modules $U g \otimes_{U b} W,\left(U g \otimes_{U b} W\right)^{*}$ are infinite-dimensional, Theorems $4.15,4.19$ show that the cohomology is always finite-dimensional.

Using Theorem 4.19 in conjunction with a certain spectral sequence we obtain a vanishing theorem, Theorem 5.9, for the cohomology of a Verma module tensored with a finite-dimensional module.

In $\$ 6$ we present a simple counterexample which shows that a result claimed by $\mathrm{H}$. Kimura in [13] is false.

We wish to express special thanks to Professor Bertram Kostant for calling to our attention certain formulas in Cartan-Eilenberg [3] (which we have used here decisively) and for rendering generous advice during various stages of this work.

2. Verma modules. Throughout this paper $g$ will denote a complex semisimple (finite-dimensional) Lie algebra. Choose a Cartan subalgebra $h$ of $g$ and let $\Delta$ denote the set of nonzero roots of $g$ relative to $h$. The Killing form (, ) is nondegenerate on $h$ so for each $\alpha$ in $\Delta$ we can choose $H_{\alpha}$ in $h$ such that

$$
\left(H, H_{\alpha}\right)=\alpha(H)
$$

for all $H$ in $h$. Let $h_{R}$ be the real vector space generated by the $H_{\alpha}, \alpha \in \Delta . h_{R}$ is a real form of $h$ and $\left.()\right|_{,h_{R}}$ is a real inner product on $h_{R}$. Relative to some lexicographic ordering on the real dual space $h_{R}^{*}$ of $h_{R}$ choose a set $\Delta^{+} \subset \Delta$ of positive roots. Let $\pi$ be a corresponding system of simple roots. We set

$$
\begin{aligned}
& n=\sum_{\alpha \in \Delta^{+}} g_{\alpha}, \quad \bar{n}=\sum_{\alpha \in \Delta^{+}} g_{-\alpha}, \\
& b=h+n, \quad \bar{b}=h+\bar{n}, \quad \delta=\frac{1}{2} \sum_{\alpha \in \Delta^{+}},
\end{aligned}
$$

where $g_{\alpha}$ is one-dimensional root space corresponding to $\alpha \in \Delta$. Then $g=h$ $+n+\bar{n}, n, \bar{n}$ are nilpotent, and $b, \bar{b}$ are Borel (i.e. maximal solvable) subalgebras of $g$. The Weyl group W of $g$ relative to $h$ is by definition the group generated by the linear transformations $\rho_{\alpha}: h_{R}^{*} \rightarrow h_{R}^{*}$ where

$$
\rho_{\alpha} \xi=\xi-\frac{2(\xi, \alpha)}{(\alpha, \alpha)} \alpha, \quad \xi \in h_{R}^{*}, \alpha \in \Delta .
$$

$\mathcal{Q}$ is in fact a finite group generated by the simple reflections $\rho_{\alpha}, \alpha \in \pi$. If $a$ is a Lie algebra $U a$ will denote its universal enveloping algebra. If $a_{1}$ is a subalgebra of $a$ we will always consider $U a_{1}$ as the subalgebra of $U a$ generated by $a_{1}, 1$.

Now let $\lambda \in h^{*}$ (complex dual space of $h$ ) be given. Then we can regard the complex number field $\mathbf{C}$ as a $b$ module: $(H+x) \cdot z=\lambda(H) z$ where $(H, x, z) \in h \times n \times \mathbf{C}$; see (2.2). Hence $\mathbf{C}$ is a left $U b$ module and we shall 
also write $\mathbf{C}=\mathbf{C}_{\lambda}$. Regarding $U g$ as a left $U g$, right $U b$ module we can form the left $U g$ module.

$$
V(\lambda)=U g \otimes_{U b} \mathbf{C}_{\lambda} .
$$

The modules $V(\lambda), \lambda \in h^{*}$, are called Verma modules [1], [14], [15]. An alternate description of $V(\lambda)$ is the following.

$$
V(\lambda)=U g /(U g)_{\lambda}
$$

where $(U g)_{\lambda}$ is the left ideal of $U g$ generated by $n$ (see (2.2)) and elements of the form $h-\lambda(H) 1, H \in h$. Indeed $(U g)_{\lambda}$ annihilates the generator (highest weight vector) $1 \otimes_{U b} 1$ of $V(\lambda)$ and thus a homeomorphism $U g /(U g)_{\lambda} \rightarrow$ $V(\lambda)$ is induced such that the coset of 1 maps to $1 \otimes_{U b} 1$. Using the PBW (Poincare-Birkhoff-Witt) theorem one sees that this map is 1-1. Moreover it is clearly a $U g$ map so that $V(\lambda), U g /(U g)_{\lambda}$ are isomorphic as $U g$ modules.

We shall also be interested in the contragredient action of $g$ on the dual $V(\lambda)^{*}$ of $V(\lambda)$ :

$$
(x \cdot f)(v)=-f(x \cdot v)
$$

for $(x, f, v) \in g \times V(\lambda)^{*} x V(\lambda)$. The character $\lambda$ of $b$ extends to an algebra homomorphism $\lambda: U b \rightarrow \mathbf{C}$. Let $\operatorname{Hom}_{U b}\left(U g, \mathbf{C}_{-\lambda}\right)$ be the space of linear maps $f: U g \rightarrow \mathbf{C}$ such that

$$
f(B A)=-\lambda(B) f(A)
$$

for $(B, A) \in U b \times U g . \operatorname{Hom}_{U b}\left(U g, \mathbf{C}_{-\lambda}\right)$ is a left $U g$ module where $U g$ acts by right translation.

Proposition 2.8. $V(\lambda)^{*}$ and $\operatorname{Hom}_{U b}\left(U g, \mathrm{C}_{-\lambda}\right)$ are isomorphic as $U g$ modules; see (2.4).

Proof. See Proposition 5.5.4 [6].

3. A duality theorem. Let $a$ be an arbitrary Lie algebra over a field $F$ of characteristic zero. Let $U, V$ be $a$ modules. Choose a $U a$ projective resolution

$$
\ldots \rightarrow U_{k} \rightarrow U_{k-1} \rightarrow \cdots \rightarrow U_{1} \rightarrow U_{0} \rightarrow U \rightarrow 0
$$

of $U$. Then we obtain maps $d_{k}$

$$
\ldots \rightarrow \operatorname{Hom}_{U a}\left(U_{k}, V\right) \rightarrow \operatorname{Hom}_{U a}\left(U_{k+1}, V\right) \rightarrow \ldots
$$

such that $d_{k} d_{k-1}=0$. By definition $\operatorname{Ext}_{U a}(U, V)$ is the cohomology of the complex $\operatorname{Hom}_{U a}\left(U_{*}, V\right)$; i.e.

$$
\operatorname{Ext}_{U a}^{k}(U, V)=\operatorname{ker} d_{k} / \operatorname{Im} d_{k-1} .
$$

The groups $\operatorname{Ext}_{U a}^{k}(U, V)$ are well defined, being independent of the choice of resolution because any two projective resultions are homotopically equivalent. For $U=F$ with $a$ acting trivially we have

$$
H^{k}(a, V) \stackrel{\text { def }}{=} \operatorname{Ext}_{U a}^{k}(F, V) .
$$


$H^{k}(a, V)$ is the $k$ th dimensional cohomology group of a with coefficients in $V$. Now $F$ admits a "standard" $U a$ projective resolution: viz.

$$
\cdots \rightarrow U a \otimes_{F} \Lambda^{k} a^{d_{k}} \rightarrow \cdots \rightarrow U a \otimes_{F} \Lambda^{0} a \stackrel{e}{\rightarrow} F
$$

where $\varepsilon$ is the augmentation epimorphism. The differential $d_{k}$ is given by $d_{k}\left(1 \otimes x_{1} \wedge \cdots \wedge x_{k}\right)$

$$
\begin{aligned}
&= \sum_{i=1}^{k}(-1)^{i+1} x_{i} \otimes x_{1} \wedge \cdots \wedge \hat{x}_{i} \wedge \cdots \wedge x_{k} \\
&+\sum_{i<j}(-1)^{i+j} 1 \otimes\left[x_{i}, x_{j}\right] \wedge x_{1} \wedge \cdots \wedge \hat{x}_{i} \\
& \wedge \cdots \wedge \hat{x}_{j} \wedge \cdots \wedge x_{k}
\end{aligned}
$$

for $x_{j}$ in $a$. In view of (3.2) the definition of $H^{k}(a, V)$ given in (3.1) coincides with that given by Chevalley-Eilenberg in [4].

Dual to the groups in (3.1) are the $k$ th dimensional homology groups $H_{k}(a, V)$ of a with coefficients in a right Ua module $V$, defined by

$$
H_{k}(a, V) \stackrel{\text { def }}{=} \operatorname{Tor}_{k}^{U a}(V, F) \text {; }
$$

see [3]. We remark that because the universal enveloping algebra $U a$ admits an antipodism one can always convert left $\mathrm{Ua}$ modules $\mathrm{V}$ into right $\mathrm{Ua}$ modules by setting $v \cdot x=-x \cdot v,(x, v) \in a \times V$.

Now we present a basic duality theorem which is valid for arbitrary (i.e. infinite-dimensional) modules. This theorem is based on duality formulas in [3].

THEOREM 3.4 (DuALITY). Let a be a finite-dimensional Lie algebra over a field $F$ of characteristic zero and let $V$ be a left Ua module. Let $V^{*}$ denote the dual space of $V$ so that $V^{*}$ is a left a module via the contragredient action given in (2.6). Then for each $k \geqslant 0$ we have a vector space isomorphism

$$
H^{k}(a, V)^{*} \simeq H^{n-k}\left(a, \Lambda^{n} a \otimes V^{*}\right)
$$

where $n=\operatorname{dim}_{F} a$ and where $\Lambda^{n} a$ is regarded as a left a module via the adjoint action.

$$
x \cdot\left(y_{1} \wedge \cdots \wedge y_{n}\right)=\sum_{i=1}^{n} y_{1} \wedge \cdots \wedge\left[x, y_{i}\right] \wedge \cdots \wedge y_{n}
$$

for $x, y_{i}$ in $a$; i.e. $x$ is scalar multiplication by trace of $\operatorname{ad}(x)$. In particular if a is unimodular (that is trace $\operatorname{ad}(x)=0$ for all $x$ in a) then for each $k>0$

$$
H^{k}(a, V)^{*} \simeq H^{n-k}\left(a, V^{*}\right) \text {. }
$$

We note that (3.6) holds if $a$ is semisimple (or even if $a$ is even reductive). Also (3.6) holds if $a$ is nilpotent. The proof of Theorem 3.4 is as follows. The 
equation

$$
(f \cdot x)(v)=f(x \cdot v)
$$

$(x, f, v) \in a \times V^{*} \times V$ defines a right module structure on $V^{*}$ (cf. (2.6)). Then taking $C^{\prime}=\mathrm{C}, A=\mathrm{C}$ in Proposition 3.3, p. 211, of [3] we get

$$
\left(\operatorname{Ext}_{U a}^{k}(\mathrm{C}, V)\right)^{*}=\operatorname{Tor}_{k}^{U a}\left(V^{*}, \mathrm{C}\right)=H_{k}\left(a, V^{*}\right) \text {; }
$$

see (3.3). Thus by (3.1)

$$
H^{k}(a, V)^{*}=H_{k}\left(a, V^{*}\right) \text {. }
$$

On the other hand if $W$ is any left $a$ module and $\tilde{W}$ is the corresponding right $a$ module induced by the antipodism of $U a$ (see remark following (3.3)) then

$$
H^{n-k}\left(a, \Lambda^{n} a \otimes W\right)=H_{k}(a, \tilde{W})
$$

by [3, p. 288], where $n=\operatorname{dim} a$.

Taking $W=V^{*}$ with the left module structure given by (2.6) we get $\tilde{W}=V^{*}$ with the right module structure given in (3.7). Thus by (3.9), (3.10) we get

$$
H^{k}(a, V)^{*}=H^{n-k}\left(a, \Lambda^{n} a \otimes V^{*}\right)
$$

as desired.

4. Cohomology computations. In this section we apply the duality theorem and key results of Kostant [12] to obtain the cohomology of a Verma module and its dual. We shall also make the Hochschild-Serre spectral sequence [9]. The starting point is a Lie-algebraic version of Shapiro's Lemma which in classical finite theory relates the cohomology of a module with that of the induced module. The precise result needed is the following.

THEOREM 4.1. Let $a$ be $a$ Lie algebra over $a$ field $F$ and let $a_{1}$ be a subalgebra of $a$. If $W$ is a left $a_{1}$ module then

$$
H^{k}\left(a_{1}, W\right) \simeq H^{k}\left(a, \operatorname{Hom}_{U a_{1}}(U a, W)\right) .
$$

See Proposition 4.2 of $[3$, p. 275].

LEMMA 4.2. Let $a$ be an abelian Lie algebra and let $W$ be an a module such that $A \cdot w=\mu(A) w$ for all $A$ in $a$ and $w$ in $W$, where $\mu \in a^{*}$. Then $H^{k}(a, W)$ $=0$ for all $k \geqslant 0$ if $\mu \neq 0$. If $\mu=0, H^{k}(a, W)=\operatorname{Hom}\left(\Lambda^{k} a, W\right)$ for all $k>0$.

Lemma 4.2 follows from a general result of Dixmier [7]. However a proof for the special case at hand is rather immediate; see [8]. Now let $\lambda \in h^{*}$ be arbitrary. Then by Theorem 4.1

$$
H^{k}\left(g, \operatorname{Hom}_{U b}\left(U g, \mathbf{C}_{\lambda}\right)\right) \simeq H^{k}\left(b, \mathbf{C}_{\lambda}\right) ;
$$


see $\$ 2$ for all notation. Since $n$ is an ideal in $b$ given any $b$ module $W$ there is a Hochschild-Serre spectral sequence $\left\{E_{r}^{p, q}\right\}$ whose $E_{\infty}$ term is associated to $H^{*}(b, W)$ and whose second order terms are given by

$$
E_{2}^{p, q}=H^{p}\left(b / n, H^{q}(n, W)\right) \text {. }
$$

In particular we have

$$
E_{2}^{p, q}=H^{p}\left(h, H^{q}\left(n, \mathbf{C}_{\lambda}\right)\right) .
$$

Now Kostant has shown, Theorem 5.14 [12], that $H^{q}(n, \mathrm{C})$ as an $h$ module is completely reducible and decomposes as follows:

$$
\begin{aligned}
H^{q}(n, \mathbf{C}) & =\sum \mathbf{C} \bar{e}_{\sigma} \quad \text { (direct sum), } \\
\sigma \in \mho & =\text { Weyl group, } \\
l(\sigma) & =q,
\end{aligned}
$$

where $l(\sigma)$ (the "length" of $\sigma$ ) is the cardinality of the subset $\sigma \Delta^{-} \cap \Delta^{+}$of $\Delta^{+}$ and

$$
\bar{e}_{\sigma}=e_{-\alpha_{1}} \wedge \cdots \wedge e_{-\alpha_{(0)}}
$$

for $\sigma \Delta^{-} \cap \Delta^{+}=\left\{\alpha_{1}, \ldots, \alpha_{l(\sigma)}\right\}, e_{-\alpha} \in g_{-\alpha}-\{0\}, \alpha \in \Delta$. Moreover the action of $h$ on $\bar{e}_{\sigma}$ is given by

$$
H \cdot \bar{e}_{\sigma}=(\sigma \delta-\delta)(H) \bar{e}_{\sigma},
$$

$H \in h, \sigma \in W$; see (2.2). Actually (4.6) is a special case of a much more general result of Kostant. Writing

$$
H^{q}\left(n, \mathrm{C}_{\lambda}\right)=H^{q}(n, \mathrm{C}) \otimes \mathbf{C}_{\lambda}
$$

(4.6), (4.7) imply that $H^{q}\left(n, \mathrm{C}_{\lambda}\right)$ has an $h$ module decomposition

$$
\begin{aligned}
H^{q}\left(n, \mathbf{C}_{\lambda}\right) & =\sum \mathrm{C}_{\sigma} \quad \text { (direct sum) } \\
\sigma \in \mathscr{W} & =\text { Weyl group, } \\
l(\sigma) & =q
\end{aligned}
$$

where $v_{\sigma} \neq 0$ and

$$
H \cdot v_{\sigma}=(\sigma \delta-\delta+\lambda)(H) v_{\sigma}
$$

for $H$ in $h$. By (4.5), (4.9)

$$
\begin{aligned}
E \sum^{p, q} & =\sum H^{p}\left(h, \mathbf{C} v_{\sigma}\right) \quad \text { (direct sum), } \\
\sigma \in \mathscr{W} & =\text { Weyl group, } \\
l(\sigma) & =q .
\end{aligned}
$$

Hence if $\lambda+\sigma \delta-\delta \neq 0$ for every $\sigma$ in the Weyl group, Lemma 4.2, (4.10), and (4.11) imply that $E_{2}^{p, q}=0$ for every $p$ and for $q>0$ and we conclude that (using (4.5)) for every $p$ 


$$
H^{p}\left(b, \mathbf{C}_{\lambda}\right)=E \varepsilon^{p, 0}=H^{p}\left(h, \mathbf{C}_{\lambda}\right)=0 ;
$$

the last statement of equality follows from Lemma 4.2 since our hypothesis excludes $\lambda$ from being zero. Next assume $\lambda+\sigma_{0} \delta-\delta=0$ for some $\sigma_{0}$ in the Weyl group, By the regularity of $\delta, \sigma_{0}$ is necessarily unique. Hence by (4.10) if $\sigma \neq \sigma_{0}$ the action of $h$ on $\mathrm{C} v_{\sigma}$ must be nontrivial and thus $H^{p}\left(h, \mathrm{C} v_{\sigma}\right)=0$ by Lemma 4.2. In other words (4.11) implies that

$$
E_{2}^{p, q}= \begin{cases}H^{p}\left(h, \mathbf{C} v_{\sigma_{0}}\right) & \text { if } q=l\left(\sigma_{0}\right), \\ 0 & \text { if } q \neq l\left(\sigma_{0}\right) .\end{cases}
$$

Hence we have for every $p$

$$
\begin{aligned}
H^{p}\left(b, \mathbf{C}_{\lambda}\right) & =E_{2}^{p-l\left(\sigma_{0}\right), l\left(\sigma_{0}\right)}=H^{p-l\left(\sigma_{0}\right)}\left(h, \mathbf{C} v_{0}\right) \\
& =\Lambda^{p-l\left(\sigma_{0}\right)} h ;
\end{aligned}
$$

again the last statement of equality follows from Lemma 4.2 and (4.10) since $\lambda+\sigma_{0} \delta-\delta=0$. By (4.3), (4.12), (4.14), and Proposition 2.8 we have proved the following theorem which gives the cohomology of the dual of a Verma module.

THEOREM 4.15. Let $g$ be a complex semisimple Lie algebra, let $b=h+n$ be a Borel subalgebra where $h$ is a Cartan subalgebra of $g$ and $n=[b, b]($ see (2.2)), let $\lambda \in h^{*}$ be a linear functional on $h$, and let $\mathbf{C}_{\lambda}$ denote the corresponding $b$ module (hence Ub module) such that $n \cdot \mathbf{C}_{\lambda}=0$. Thus $\operatorname{Hom}_{U b}\left(U g, \mathbf{C}_{\lambda}\right)$ is a Ug module, where $U q$ acts by right translation. The cohomology of $g$ with coefficients in $\mathrm{Hom}_{U b}\left(U g, \mathbf{C}_{\lambda}\right)$ is given as follows.

1. If $\lambda \neq-(\sigma \delta-\delta)$ for every $\sigma$ in $\mho$ then

$$
H^{k}\left(g, \operatorname{Hom}_{U_{g}}\left(U g, \mathbf{C}_{\lambda}\right)\right)=0
$$

for all $k \geqslant 0$.

2. If $\lambda=-(\sigma \delta-\delta)$ for some $\sigma$ in $\mho(\sigma$ is necessarily unique) then

$$
H^{k}\left(g, \operatorname{Hom}_{U g}\left(U g, C_{\lambda}\right)\right)=\Lambda^{k-l(o)} h .
$$

Here $थ$ is the Weyl group of $g$ relative to $h, l(\sigma)$ (the length of $\sigma$ ) is the cardinality of the set $\sigma \Delta^{-} \cap \Delta^{+}, \Delta^{+}$is the set of positive roots and

$$
\delta=\frac{1}{2} \sum_{\alpha \in \Delta^{+}} \alpha
$$

see $\$ 2$. In either case

$$
H^{k}\left(g, \operatorname{Hom}_{U b}\left(U g, \mathbf{C}_{\lambda}\right)\right)=H^{k}\left(b, \mathbf{C}_{\lambda}\right) ;
$$

see (4.3). If $\lambda=-(\sigma \delta-\delta)$, as in $\S 2$, then in particular

$$
H^{k}\left(g, \operatorname{Hom}_{U b}\left(U g, \mathbf{C}_{\lambda}\right)\right)=0
$$

unless $l(\sigma)<k<\operatorname{dim}_{\mathrm{c}} h+l(\sigma)$. 
Again suppose $\lambda \in h^{*}$ is arbitrary. Applying Proposition 2.8 again we have

$$
H^{q}\left(g,\left(U g \otimes_{U b} \mathbf{C}_{\lambda}\right)^{*}\right)=H^{q}\left(g, \operatorname{Hom}_{U b}\left(U g, \mathbf{C}_{-\lambda}\right)\right)
$$

for $q>0$. On the other hand by the duality theorem (Theorem 3.4)

$$
H^{k}\left(g, U g \otimes_{U b} \mathbf{C}_{\lambda}\right)^{*}=H^{\operatorname{dim} g-k}\left(g,\left(U g \otimes_{U b} \mathbf{C}_{\lambda}\right)^{*}\right) .
$$

Thus by (4.16), (4.17)

$$
H^{k}\left(g, U g \otimes_{U b} \mathbf{C}_{\lambda}\right)^{*}=H^{\operatorname{dim} g-k}\left(g, \operatorname{Hom}_{U b}\left(U g, \mathbf{C}_{-\lambda}\right)\right) .
$$

From (4.18) and Theorem 4.15 we deduce that $H^{k}\left(g, U g \otimes_{U b} C_{\lambda}\right)$ is finite dimensional for each $k$ and moreover

THEOREM 4.19. Let $g$ be a complex semisimple Lie algebra, $h$ a Cartan subalgebra of $g, b=h+[b, b]$ a Borel subalgebra of $g$, and let W be the Weyl group of $g$ relative to $h$. For $\sigma$ in ข let $l(\sigma)$ denote the length of $\sigma$ (as in Theorem 4.15) and let

$$
\delta=\frac{1}{2} \sum_{\alpha \in \Delta^{+}} \alpha
$$

given a choice of positive roots $\Delta^{+}$. If $\lambda \in h^{*}$ is any linear functional on $h$ and $U g \otimes_{U b} \mathbf{C}_{\lambda}$ is the corresponding Verma module (see \$2) then the cohomology of $\mathbf{g}$ with $U \mathbf{g} \otimes_{U b} \mathbf{C}_{\lambda}$ coefficients is given as follows.

1. If $\lambda \neq \sigma \delta-\delta$ for every $\sigma$ in $\mho$ then

$$
H^{k}\left(g, U g \otimes_{U b} \mathbf{C}_{\lambda}\right)=0
$$

for all $k \geqslant 0$.

2. If $\lambda=\sigma \delta-\delta$ for some $\sigma$ in $W$ ( $\sigma$ is necessarily unique) then

$$
H^{k}\left(g, U g \otimes_{U b} C_{\lambda}\right)=\Lambda^{\operatorname{dim} g-k-l(o)} h .
$$

Let $l=\operatorname{dim}_{\mathrm{C}} h=$ rank of $g$ and let $p=\left|\Delta^{+}\right|=$number of positive roots. If $\lambda=\sigma \delta-\delta$, as in 2 , then in particular

$$
H^{k}\left(g, U g \otimes_{U b} \mathbf{C}_{\lambda}\right)=0
$$

unless $2 p-l(\sigma)<k<l+2 p-l(\sigma)=\operatorname{dim} g-l(\sigma)$. Thus for Verma modules there is no "low-dimensional" cohomology. Moreover

$$
H^{*}\left(g, U g \otimes_{U b} \mathbf{C}_{\lambda}\right) \stackrel{\text { def }}{=} \sum_{k=0}^{\operatorname{dim} g} H^{k}\left(g, U g \otimes_{U b} \mathbf{C}_{\lambda}\right) \quad \text { (direct sum) }
$$

has dimension equal to $2^{l}$.

COROLlaRY 4.20. If $\lambda=\sigma \delta-\delta$ then for each simple root $\alpha \in \pi$ such that $l\left(p_{\alpha} \sigma\right)=l(\sigma)-1$ one has

$$
H^{k}\left(g, U g \otimes_{U b} \mathbf{C}_{\lambda}\right)=H^{k+1}\left(g, U g \otimes_{U b} \mathbf{C}_{\rho_{\alpha} \lambda-\alpha}\right) .
$$


Here $\rho \alpha \in \mathscr{W}$ is the simple Weyl reflection defined by $\alpha$; hence $\rho_{\alpha} \lambda-\alpha=$ $\rho_{\alpha}(\lambda+\delta)-\delta$; see (2.3).

Proof. Since $\alpha$ is simple $2(\delta, \alpha) /(\alpha, \alpha)=1$ so that $\rho_{\alpha} \lambda-\alpha=\rho_{\alpha}(\sigma \delta-\delta)$ $-\alpha=\rho_{\alpha} \sigma \delta-\delta$ by (2.3) (taking $\xi=\delta$ ). Thus by Theorem 4.19

$$
\begin{aligned}
H^{k+1}\left(g, U g \otimes_{U b} \mathbf{C}_{\rho_{\alpha} \lambda-\alpha}\right) & =\Lambda^{\operatorname{dim} g-(k+1)-l\left(\rho_{\alpha} \sigma\right)} h \\
& =\Lambda^{\operatorname{dim} g-k-l(\sigma)} h=H^{k}\left(g, U g \otimes_{U b} \mathbf{C}_{\lambda}\right)
\end{aligned}
$$

since $l\left(\rho_{\alpha} \sigma\right)=l(\sigma)-1$ by hypothesis.

Clearly formula (4.21) is quite similar in form to the "dimension shifting" formula of Bott [2] (or Demazure [5]) for the sheaf cohomology of certain line bundles in connection with the Borel-Weil Theorem.

5. The spectral sequence induced by filtered submodules. The tensor product of a Verma module with a finite-dimensional module always has a finite decreasing filtration by submodules such that the corresponding subquotients are Verma modules. Using such a filtration we construct a spectral sequence whose first order terms $E_{1}^{p, q}$ are the $p+q$ dimensional cohomology of Verma modules. Applying Theorem 4.19 we obtain a vanishing theorem, Theorem 5.9 , for the cohomology of the tensor product.

Let $a$ be a Lie algebra and let $V$ be an $a$ module. Suppose $V$ has a decreasing filtration by submodules $\left\{V_{p}\right\}_{p \in Z}$ ( $Z$ is the set of integers):

$$
\ldots V_{-1} \supset V_{0} \supset V_{1} \supset V_{2} \supset \ldots
$$

Then there is an induced decreasing filtration $\left\{\operatorname{Hoin}\left(\Lambda^{k} a, V\right)_{p}\right\}_{p \in Z}$ of $\operatorname{Hom}\left(\Lambda^{k} a, V\right)$ such that

$$
d_{k} \operatorname{Hom}\left(\Lambda^{k} a, V\right)_{p} \subset\left\{\operatorname{Hom}\left(\Lambda^{k+1} a, V\right)_{p}\right\}_{p}
$$

for each $k$ and $p$; see (3.5). Put

$$
\Lambda(a, V)=\sum_{k} \operatorname{Hom}\left(\Lambda^{k} a, V\right) \quad \text { (direct sum). }
$$

Then because of (5.1) we can define a decreasing filtration $\left\{\Lambda(a, V)_{p}\right\}_{p \in Z}$ on the cochain complex $(\Lambda(a, V), d)$ (i.e. a filtration $\left\{\Lambda(a, V)_{p}\right\}_{p \in Z}$ such that $\left.d \Lambda(a, V)_{p} \subset \Lambda(a, V)_{p}\right)$ by setting

$$
\Lambda(a, V)_{p}=\sum_{k} \operatorname{Hom}\left(\Lambda^{k} a, V\right)_{p} \quad \text { (direct sum). }
$$

Moreover this filtration is compatible with the grading of $\Lambda(a, V)$. Thus $\left\{V_{p}\right\}_{p \in Z}$ induces a spectral sequence $\left\{E_{r}^{p, q}\right\}$ whose $E_{\infty}$ term is associated with the cohomology of the complex $(\Lambda(a, V), d)$. We have

$$
E_{0}^{p, q}=\operatorname{Hom}\left(\Lambda^{p+q} a, V\right)_{p} / \operatorname{Hom}\left(\Lambda^{p+q} a\right)_{p+1} .
$$

We shall consider elements in $\operatorname{Hom}\left(\Lambda^{k} a, V\right)$ as alternate $k$ linear maps from $a^{k}$ to $V$. There are natural isomorphisms: 


$$
\operatorname{Hom}\left(\Lambda^{k} a, V\right)_{p} / \operatorname{Hom}\left(\Lambda^{k} a, V\right)_{p+1} \simeq \operatorname{Hom}\left(\Lambda^{k} a, V_{p} / V_{p+1}\right) .
$$

Moreover the diagrams

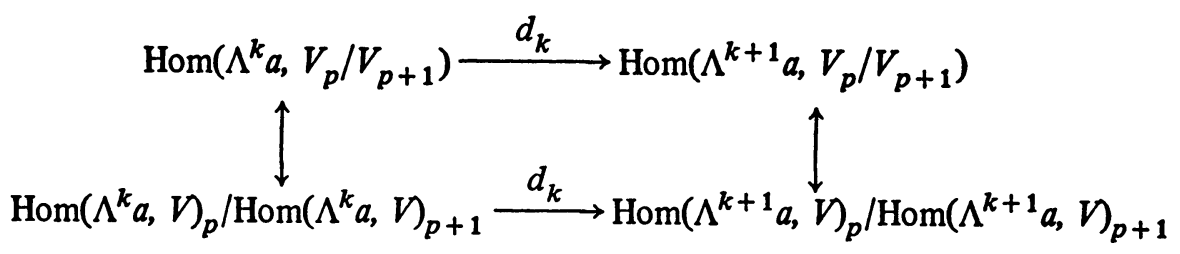

are commutative. Keeping (5.4) in mind we can therefore conclude

THEOREM 5.5. If $a$ is a Lie algebra and $V$ is an a module with a decreasing filtration by submodules $\left\{V_{p}\right\}_{p \in Z}$ then there is a spectral sequence $\left\{E_{r}^{p, q}\right\}$ such that $E_{\infty}$ is associated with $H^{*}(a, V)$ and

$$
E_{1}^{p, q}=H^{p+q}\left(a, V_{p} / V_{p+1}\right) \text {. }
$$

The filtered complex $\left\{\Lambda(a, V)_{p}, d\right\}$ will certainly be regular if we assume, in particular, that $V_{p}=V$ for $p<0$ and $V_{p}=0$ for $p$ sufficiently large. These assumptions are satisfied in the following situation. Let $V(\lambda)=U g \otimes_{U b} \mathbf{C}_{\lambda}$ be a Verma module (as in (2.4)) and let $V$ be any finite-dimensional $g$ module. Then the tensor product $V(\lambda) \otimes V$ admits a decreasing filtration

$$
V(\lambda) \otimes V=V_{0} \supset V_{1} \supset \cdots \supset V_{n}=0
$$

by submodules $\left\{V_{p}\right\}_{p}^{n=\operatorname{dim} V}$ such that

$$
V_{p} / V_{p+1} \simeq V\left(\lambda+\mu_{p}\right)
$$

where $\mu_{p}$ is a weight of $V$ and $V\left(\lambda+\mu_{p}\right)$ is the Verma module defined by $\lambda+\mu_{p} \in h^{*}$; see [1], [13]. Hence by Theorem 5.5 there is a spectral sequence such that

$$
E_{1}^{p, q}=H^{p+q}\left(g, V\left(\lambda+\mu_{p}\right)\right)
$$

and such that $E_{\infty}$ is associated with $H^{*}(g, V(\lambda) \otimes V)$. If for every $\sigma$ in $\mho$ and for every weight $\mu$ of $V, \lambda \neq \sigma \delta-\delta-\mu$ then $\lambda+\mu_{p} \neq \sigma \delta-\delta$ for each $p$ and $E p^{p, q}=0$ for each $p, q$ by (5.8) and Theorem 4.19. Hence for every $p$

$$
H^{p}(g, V(\lambda) \otimes V)=E_{1}^{0, p}=0
$$

and we have proved

Theorem 5.9. Let $g, h, b$, ข $\delta$ be as in Theorem 4.19 , let $\lambda \in h^{*}$, and let $V$ be any finite-dimensional $g$ module. Then

$$
H^{k}\left(g,\left(U g \otimes_{U b} \mathbf{C}_{\lambda}\right) \otimes V\right)=0
$$

for all $k>0$ unless $\lambda$ has the form $\lambda=\sigma \delta-\delta-\mu$ for some $\sigma$ in $W$ and some weight $\mu$ of $V$. 
6. A counterexample. H. Kimura asserts in Theorem 2 of [13] that if $V$ is a $g$ module generated by a highest weight vector and having $\lambda$ as its highest weight then

$$
H^{1}(g, V)= \begin{cases}C & \text { if } \lambda=-\alpha, \\ 0 & \text { otherwise, }\end{cases}
$$

where $\alpha$ is a simple root. We shall show by a simple example that this assertion is false. More precisely if $g=\operatorname{sl}(3, \mathrm{C})$ and if $V$ is a Verma module with highest weight $\lambda=-\alpha, \alpha$ a simple root, then we claim

$$
H^{1}(g, V)=0 \text {. }
$$

Indeed we remarked in Theorem 4.19 that for Verma modules the cohomology in low dimensions vanishes. In particular, by Theorem 4.19, $H^{k}(g, V)=0$ unless $k \geqslant$ number of positive roots. Thus Theorem 4.19 implies (6.2). However we shall give a direct proof of (6.2)-one which is therefore independent of Theorem 4.19. We remark that part of Kimura's statement is true. Namely if $-\lambda \neq$ each simple root then $H^{1}(g, V)=0$.

Now we assume $g$ is the Lie algebra sl $(3, \mathrm{C})$ of $3 \times 3$ complex matrices of trace zero. There are two simple roots $\alpha_{1}, \alpha_{2}$ :

$$
\Delta^{+}=\left\{\alpha_{1}, \alpha_{2}, \alpha_{3}\right\}, \quad \pi=\left\{\alpha_{1}, \alpha_{2}\right\},
$$

where $\alpha_{3}=\alpha_{1}+\alpha_{2}$. Let $x_{j}, y_{j}$ denote generators of the one-dimensional root spaces (for nonzero roots) so that

$$
g_{\alpha_{j}}=\mathbf{C} x_{j}, \quad g_{-\alpha_{j}}=\mathbf{C} y_{j},
$$

$\alpha_{j} \in \Delta^{+}$. We may assume

$$
\left[x_{1}, x_{2}\right]=x_{3}, \quad\left[y_{1}, y_{2}\right]=y_{3} .
$$

Proposition 6.6. Suppose $V=V(\lambda)$ is a Verma module for $g=\mathrm{sl}(3, \mathrm{C})$ with highest weight $\lambda=-\alpha$, where $\alpha$ is a simple root. Then 0 is not a weight of $V$, no positive root is a weight of $V$, and $-\beta$ is not a weight of $V$ if $\beta$ is a simple root $\neq \lambda=-\alpha$.

The proof follows since every weight of $V$ has the form $-\alpha-n_{1} \alpha_{1}-n_{2} \alpha_{2}$ for suitable nonnegative integers $n_{1}, n_{2}$. We consider $V$ as in Proposition 6.6. To establish (6.2) we must show the following: If $w \in \operatorname{Hom}(g, V)$ is any 1-cocycle, i.e.

$$
w([x, y])=x \cdot w(y)-y \cdot w(x)
$$

for all $x, y$ in $g$, then $w$ is trivial; i.e. there exists a $v$ in $V$ such that

$$
w(x)=x \cdot v
$$

for all $x$ in $g$. Thus suppose $w \in \operatorname{Hom}(g, V)$ is given which satisfies (6.7). Considering $V$ as an $h$ module ( $h$ a Cartan subalgebra) one has

$$
H^{1}(h, V)=0 \text {. }
$$


Indeed

where

$$
H^{1}(h, V)=\sum_{\mu} H^{1}\left(h, V_{\mu}\right) \quad \text { (direct sum) }
$$

$$
V=\sum V_{\mu}, \quad \mu=\text { weight of } V,
$$

is a direct sum decomposition of $V$ into weight spaces $V_{\mu}$. Since 0 is not a weight of $V$, by Proposition 6.6, (6.8) follows from (6.9) and Lemma 4.2; cf. Corollary to Lemma 1 of [8].

Now $\left.w\right|_{h}$ is a 1-cocycle for $V$ as an $h$ module so by (6.8) $\left.w\right|_{h}$ is trivial; i.e. there exists a $v$ in $V$ such that

$$
w(H)=H \cdot V
$$

for all $H$ in $h$. Let $\alpha \in \Delta$ be any nonzero root, let $x \in g_{\alpha}$, and let $H \in h$ be arbitrary. Then by (6.7)

$$
\alpha(H) w(x)=w([H, x])=H \cdot w(x)-x \cdot w(H)
$$

and by $(6.10)$

$$
\begin{aligned}
x \cdot w(H)=x \cdot(H \cdot v) & =[x, H] \cdot v+H \cdot(x \cdot v) \\
& =-\alpha(H) x \cdot v+H \cdot(x \cdot v)
\end{aligned}
$$

so that (6.11), (6.12) imply

$$
\alpha(H) w(x)=H \cdot w(x)+\alpha(H) x \cdot v-H \cdot(x \cdot v) ;
$$

i.e.,

$$
H \cdot(w(x)-x \cdot v)=\alpha(H)(w(x)-x \cdot v)
$$

for $H \in h, x \in g_{\alpha}, \alpha \in \Delta$. If $\alpha \in \Delta^{+}$in particular then by Proposition $6.6 \alpha$ is not a weight. Thus by 6.13 we must have

$$
w\left(x_{j}\right)=x_{j} \cdot v, \quad j=1,2,3 ;
$$

see (6.4). To be specific assume $\lambda=-\alpha_{1}$. Then $-\alpha_{2}$ is not a weight by Proposition 6.6 and, similarly, (6.13) implies (see (6.4))

$$
w\left(y_{2}\right)=y_{2} \cdot v \text {. }
$$

The highest weight space of $V$ is one-dimensional and is spanned by a nonzero vector, say, $v_{\lambda}$. (6.13) implies that $w\left(y_{1}\right)-y_{1} \cdot v\left(y_{1} \in g_{-\alpha_{1}}\right.$ by (6.4)) is in the highest weight space and therefore

$$
w\left(y_{1}\right)-y_{1} \cdot v=c v_{\lambda}
$$

for some complex number $c$. By (6.5), (6.7), (6.15), and (6.16)

$$
\begin{aligned}
w\left(y_{3}\right) & =w\left(\left[y_{1}, y_{2}\right]\right)=y_{1} \cdot w\left(y_{2}\right)-y_{2} \cdot w\left(y_{1}\right) \\
& =y_{1} \cdot\left(y_{2} \cdot v\right)-y_{2} \cdot\left(y_{1} \cdot v+c v_{\lambda}\right) \\
& =y_{3} \cdot v-c y_{2} \cdot v_{\lambda} .
\end{aligned}
$$


Since $\left[y_{2}, y_{3}\right]=0,(6.7),(6.15)$ and (6.17) imply

$$
\begin{aligned}
0 & =w\left(\left[y_{2}, y_{3}\right]\right)=y_{2} \cdot w\left(y_{3}\right)-y_{3} \cdot w\left(y_{2}\right) \\
& =y_{2} \cdot\left(y_{3} \cdot v-c y_{2} \cdot v_{\lambda}\right)-y_{3} \cdot\left(y_{2} \cdot v\right)=\left[y_{2}, y_{3}\right] \cdot v-c y_{2}^{2} \cdot v_{\lambda} \\
& =-c y_{2}^{2} \cdot v_{\lambda} .
\end{aligned}
$$

But for a Verma module $U \bar{n}$ (see (2.2)) acts without zero divisors. Hence $c=0$ and (6.16), (6.17) imply

$$
w\left(y_{1}\right)=y_{1} \cdot v, \quad w\left(y_{3}\right)=y_{3} \cdot v .
$$

Clearly (6.10), (6.14), (6.15) and (6.18) imply (6.7) and we have thus proved (6.2) for $\lambda=-\alpha_{1}$. The case $\lambda=-\alpha_{2}$ is entirely similar.

The above example can be generalized, of course, and much of our arguments remain valid up to a point for any cyclic module with a highest weight. However the crucial property of Verma modules which we exploited was the absence of zero divisors with respect ot the $U \bar{n}$ action.

\section{REFERENCES}

1. I. N. Bernštein, I. M. Gel'fand and S. I. Gel'fand, The structure of representations generated by highest weight vectors, Funkcional. Anal. i Priložen. 5 (1971), no. 1, 1-9 = Functional Anal. Appl. 5 (1971), 1-8. MR 45 \#298.

2. R. Bott, Homogeneous vector bundles, Ann. of Math. (2) 66, (1957), 203-248. MR 19, 681.

3. H. Cartan and S. Eilenberg, Homological algebra, Princeton Univ. Press, Princeton, N. J., 1956. MR 17, 1040.

4. C. Chevalley and S. Eilenberg, Cohomology theory of Lie groups and Lie algebras, Trans. Amer. Math. Soc. 63 (1948), 85-124. MR 9, 567.

5. M. Demazure, Une démonstration algébrique d'un théorème de Bott, Invent. Math. 5 (1968) 349-356. MR 37 \# 4831.

6. J. Dixmier, Algèbres enveloppantes, Gautier-Villars, Paris, 1974.

7. Cohomologie des algèbres de Lie nilpotents, Acta Sci. Math. Szeged 16 (1955), 246-250. MR 17, 645.

8. A. Hattori, On 1-cohomology groups of infinite dimensional representations of semisimple Lie algebras, J. Math. Soc. Japan 16 (1964), 226-229. MR 31 \#5930.

9. G. Hochschild and J. P. Serre, Cohomology of Lie algebras, Ann. of Math. (32) 57 (1953), 591-603. MR 14, 943.

10. J. E. Humphreys, Introduction to Lie algebras and representation theory, Springer-Verlag, Berlin and New York, 1972. MR 48 \#2197.

11. N. Jacobson, Lie algebras, Interscience Tracts in Pure and Appl. Math., no. 10, Interscience, New York, 1962. MR 26 \# 1345.

12. B. Kostant, Lie algebra cohomology and the generalized Borel-Weil theorem, Ann. of Math. (2) 74 (1961), 329-387. MR 26 \#265.

13. H. Kimura, On some infinite dimensional representations of semisimple Lie algebras, Nagoya Math. J. 25 (1965), 211-220. MR 31 \#5933.

14. D.-N. Verma, Structure of certain induced representations of complex semisimple Lie algebras, Bull. Amer. Math. Soc. 74 (1968), 160-166. MR 36 \# 1503.

15. __ Ph.D. Thesis, Yale Univ., 1966.

Department of Mathematics, Untversity of Massachusetts, Amherst, Massachusetts 01003 